NOTES

\title{
Synthesis of Temperature-Responsive Organic-Inorganic Hybrid Hydrogel by Free-Radical Polymerization of Methacrylamide Using Water-Soluble Rigid Polysiloxane Having Acryamido Side-Chains as a Cross-linking Agent
}

\author{
Yoshiro KaneKo, Katsuhiro NogUCHI, and Jun-ichi KAdOKAwA ${ }^{\dagger}$ \\ Department of Nano-structured \& Advanced Materials, Graduate School of Science and Engineering, \\ Kagoshima University, 1-21-40 Korimoto, Kagoshima 890-0065, Japan
}

(Received May 1, 2007; Accepted June 23, 2007; Published August 7, 2007)

\begin{abstract}
KEY WORDS Organic-Inorganic Hybrid / Temperature-Response / Hydrogel / Water-Soluble Polysiloxane / Polymethacrylamide / Free-Radical Polymerization / [doi:10.1295/polymj.PJ2007028]
\end{abstract}

Polymeric hydrogels are three-dimensional polymer networks containing a large amount of water, which have been used in a variety of applications, e.g., soft contact lenses, absorbents, and cosmetics, due to their high water contents and soft touchness. ${ }^{1}$ In particular, a considerable number of researches have been focused on stimuli-responsive hydrogels, in which they can change their properties in response to environmental stimuli such as $\mathrm{pH}$, temperature, ionic strength, and electric field. ${ }^{2,3}$ The stimuli-responsive hydrogels have been investigated for many biomedical and pharmaceutical applications, e.g., drug delivery system (DDS). ${ }^{2,3}$ As familiar examples, it has been reported that the hydrogels composed of poly( $N$-isopropylacrylamide), poly( $N, N$-diethylacrylamide), and poly(ethylene oxide)-poly(propylene oxide) block copolymer have exhibited temperature-response. ${ }^{2-6}$

On the other hand, the hydrogels had some serious disadvantages such as brittleness and lack of mechanical toughness. So far, to solve these disadvantages of the hydrogels, synthesis of organic-inorganic nanocomposite (hybrid) hydrogels have been reported, ${ }^{7,8}$ which are composed of organic polymer as a mainchain and inorganic dispersed clay as a cross-linking agent. A clay has two-dimensional rigid sheet structure forming regular higher-ordered structure (lamellar phase) and can disperse in the water. These properties of the clay would contribute to the superior characteristics such as high mechanical strength, elongation, and transparency of the hydrogels. However, since other inorganic materials, which have rigid structure and dispersion behavior, have hardly ever been known, the variety of the organic-inorganic hybrid hydrogels has been restricted. The availability of new inorganic cross-linking agents promisingly introduces additional development of new functions and applications of novel high-performance hybrid hydrogels.

So far, water-soluble polysiloxanes having alkylammonium groups were synthesized by the sol-gel reaction of aminoalkyltrialkoxysilane in strong acid aqueous solution. ${ }^{9,10}$ In the solid state, these polysiloxanes were stacked to form the hexagonal phase, implying their rigid-rod structures. The polysiloxanes have inspired us to use them for the development of a new hybrid hydrogel, because of their comparable water-dispersibility and rigidity to those of a clay. Accordingly, we prepared a polysiloxane-based multifunctional cross-linking agent for a new organic-inorganic hybrid hydrogel. ${ }^{11}$ The cross-linking agent was synthesized by reaction of the rigid polysiloxane with acryloyl chloride. Then, the organic-inorgnic hybrid hydrogels were obtained by free-radical polymerization of an acrylamide (AAm) monomer using the cross-linking agent. The compressive properties of the obtained hydrogels were more superior than those of conventional poly(AAm) (PAAm) hydrogels. Based on the backgrounds described above, development of novel temperature-responsive organic-inorganic hybrid hydrogels would be a promising topic in the material research fields.

In a series of the study, we prepared a polymethacrylamide (PMAAm)-type organic-inorganic hybrid hydrogel using the cross-linking agent mentioned above. We found that the new organic-inorganic hybrid hydrogel appeared temperature-responsive property, although PMAAm does not generally exhibit temperature-responsive behavior. Appropriate balance

${ }^{\dagger}$ To whom correspondence should be addressed (Tel: +81-99-285-7743, Fax: +81-99-285-3253, E-mail: kadokawa@eng.kagoshima-u.ac.jp). 


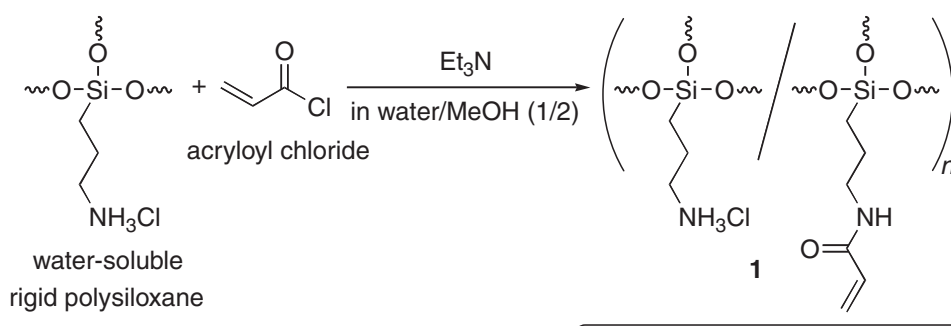

functionality of acrylamido group

1a: $8 \%, 1 b: 17 \%, 1 c: 21 \%$

Scheme 1. Synthesis of cross-linking agents 1 using water-soluble rigid polysiloxane.

between methacrylamide (MAAm) monomer and the polysiloxane-based cross-linking agent probably affects such a behavior. In this paper, we report synthesis, temperature-responsive behavior, and mechanical property of the new organic-inorganic hybrid hydrogel.

\section{EXPERIMENTAL}

\section{Materials}

The water-soluble rigid polysiloxane was prepared according to the literature procedure. ${ }^{9}$ Its molecular weight $\left(M_{\mathrm{n}}\right)$ was evaluated to be $10,300 \mathrm{~g} / \mathrm{mol}$ by GPC analysis with water as the eluent using pullulan standards. ${ }^{12}$ The cross-linking agents (1a-c) were prepared using this water-soluble polysiloxane according to a literature procedure, ${ }^{11}$ which were achieved by reaction of amino groups of the polysiloxane with acryloyl chloride. The functionalities of acrylamido groups determined by ${ }^{1} \mathrm{H}$ NMR spectra were $8 \%$ (1a), $17 \%$ (1b), and $21 \%$ (1c). Other reagents and solvents were used as received.

\section{Synthesis of Temperature-Responsive Organic-Inor-} ganic Hybrid Hydrogels (2)

A typical experimental procedure for synthesis of temperature-responsive organic-inorganic hybrid hydrogel (2a) was as follows. A cross-linking agent 1a $(0.059 \mathrm{~g}, 0.40 \mathrm{mmol}$ unit), MAAm $(0.681 \mathrm{~g}, 8.0 \mathrm{mmol})$ and potassium persulfate (KPS) $(0.022 \mathrm{~g}, 0.080 \mathrm{mmol})$ were dissolved in degassed water $(4.0 \mathrm{~mL})$, and then oxygen was excluded from the reaction system by substitution of argon. Free-radical polymerization of this mixture was carried out by heating at $60^{\circ} \mathrm{C}$ for $5 \mathrm{~h}$ to obtain $\mathbf{2 a}$.

Synthesis of PMAAm Hydrogels (3) Using Conventional Organic Cross-Linking Agent

A typical experimental procedure for preparation of PMAAm hydrogel (3a) using conventional organic cross-linking agent was as follows. $N, N^{\prime}$-Methylenebisacrylamide (BisAAm) $(0.006 \mathrm{~g}, 0.040 \mathrm{mmol})$ as a cross-linking agent, MAAm $(0.681 \mathrm{~g}, 8.0 \mathrm{mmol})$ and
KPS $(0.022 \mathrm{~g}, 0.080 \mathrm{mmol})$ were dissolved in degassed water $(4.0 \mathrm{~mL})$, and the following procedures were same as those for synthesis of $\mathbf{2 a}$.

\section{Measurements}

The transmittances of the products were measured using a UV-160A spectrometer (Shimadzu Co., Ltd.) at the scanning rate of $400 \mathrm{~nm} / \mathrm{min}$. A cell containing water was used for the reference. The fracture stresses and strains were determined by compressive stressstrain measurements using a tensile-compressive tester (Little Senstar LSC-1/30, Tokyo Testing Machine Co.). The samples were water-swollen cylindrical gels with a diameter of $13 \mathrm{~mm}$ and a height of $15 \mathrm{~mm}$. The compressive strain rate was $1.0 \mathrm{~mm} / \mathrm{min}$.

\section{RESULTS AND DISCUSSION}

The multifunctional cross-linking agents (1a-c) were prepared according to a literature procedure, ${ }^{11}$ which were achieved by reaction of amino groups of the water-soluble rigid polysiloxane with acryloyl chloride (Scheme 1). Hybrid hydrogels (2) were synthesized by free-radical polymerization of MAAm using 1 initiated with KPS in degassed water under argon atmosphere at $60^{\circ} \mathrm{C}$ for $5 \mathrm{~h}$ (Scheme 2). For the present study, we employed three cross-linking agents (1a-c) with different functionalities of acrylamido groups; $8 \%, 17 \%, 21 \%$. Because $5 \mathrm{~mol}$ unit $\%$ of 1a-c for MAAm were employed, the ratios of acrylamido groups in 1a-c to MAAm were calculated to be 0.4 , 0.85 , and $1.05 \%$, respectively. The obtained hydrogels 2a-c contained water weighting more than $80 \%$ of the materials $(\mathbf{2} \mathbf{a} ; 82 \%, \mathbf{2 b} ; 81 \%, \mathbf{2 c} ; 80 \%)$. Use of the original water-soluble rigid polysiloxane instead of $\mathbf{1}$ did not afford a hydrogel, indicating the necessity of a chemical cross-linking agent in this study.

Influences of temperature on the transmittances in visible light (wavelength; $500 \mathrm{~nm}$ ) of hybrid hydrogels 2 and conventional hydrogels $\mathbf{3}$ during heatingcooling cycles between $25^{\circ} \mathrm{C}$ and $80^{\circ} \mathrm{C}$ are shown in Figure 1. The hybrid hydrogel 2a obtained using 1a (functionality of acrylamido group; 8\%) was turbid 


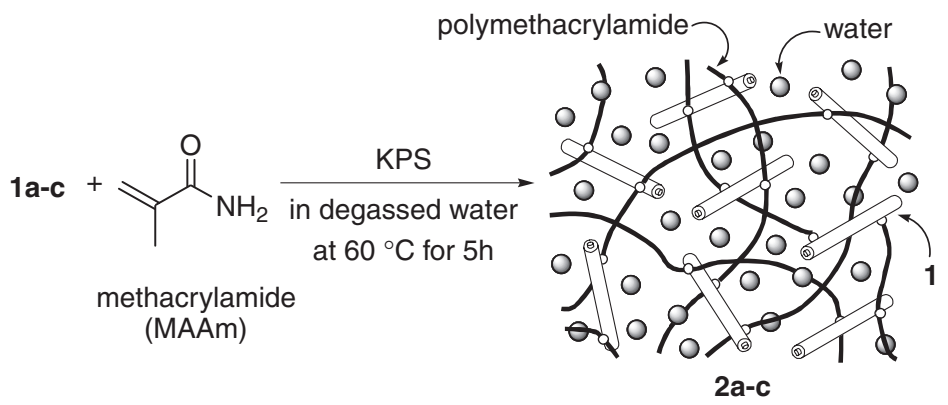

Scheme 2. Synthesis of temperature-responsive organic-inorganic hybrid hydrogels 2.
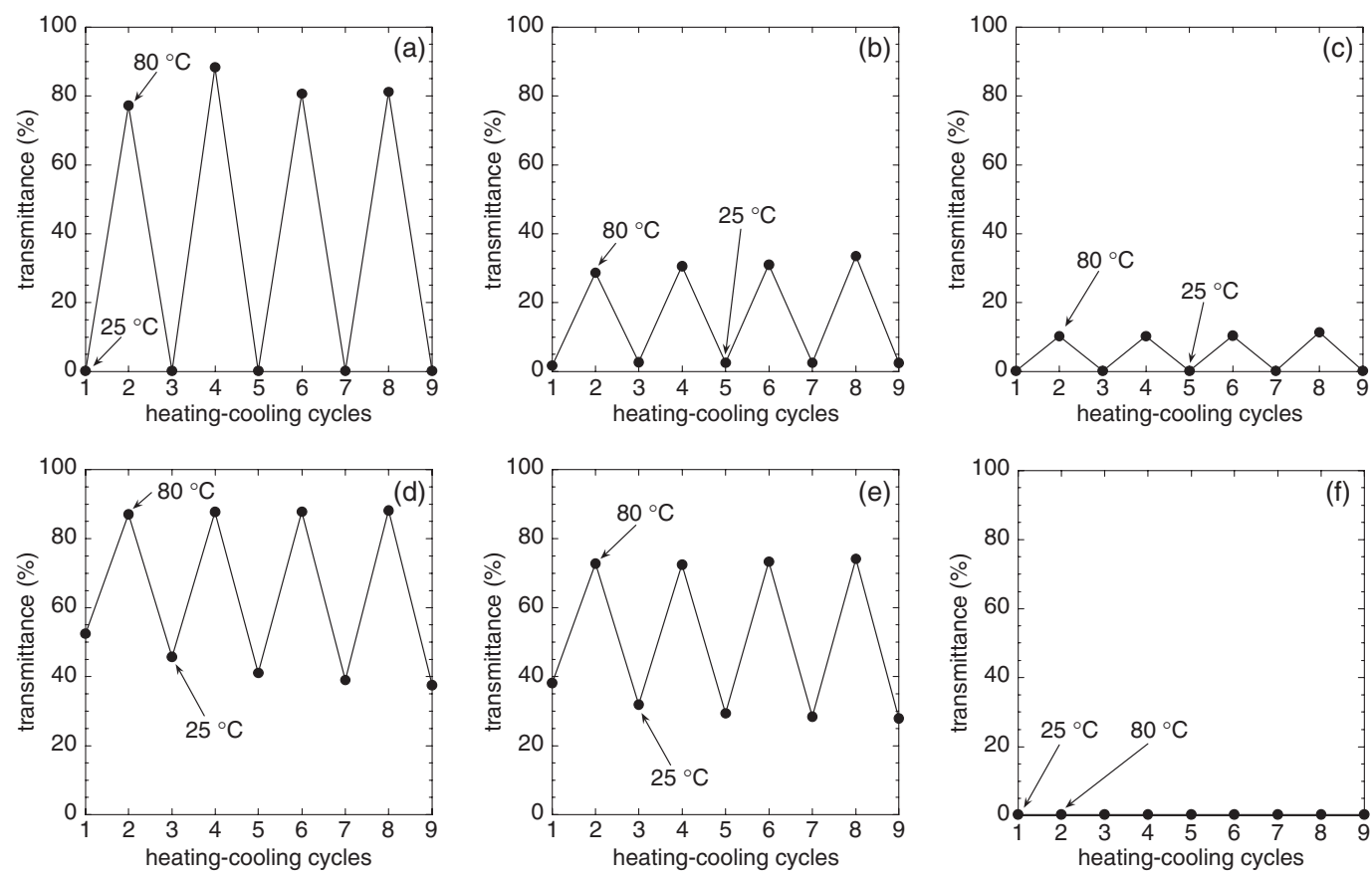

Figure 1. Transmittances in visible light (wavelength; $500 \mathrm{~nm}$ ) of hybrid hydrogel $\mathbf{2 a}(\mathrm{a}), \mathbf{2 b}(\mathrm{b}), \mathbf{2 c}(\mathrm{c})$ and hydrogels $\mathbf{3 a}(\mathrm{d}), \mathbf{3 b}(\mathrm{b})$, 3c (f) during heating-cooling cycles between $25^{\circ} \mathrm{C}$ and $80^{\circ} \mathrm{C}$.

at $25^{\circ} \mathrm{C}$ (transmittance; $0 \%$ ), while $2 \mathbf{a}$ at $80^{\circ} \mathrm{C}$ was almost transparent (transmittance; ca. 80\%) (Figure 1a). Such behaviors were observed repeatedly during heating-cooling cycles. Photographs as shown in Figure 2 also demonstrate that $\mathbf{2 a}$ has temperature-responsive property. The temperature-responsive property is probably attributed to aggregation at lower temperature and dispersion at higher temperature of the polymer chains in the hydrogels.

When 1b or 1c, which had higher functionality of acrylamido groups (17\% and $21 \%$ ) than that of $\mathbf{1 a}$, was used, the obtained hybrid hydrogels $\mathbf{2 b}$, $\mathbf{c}$ did not exhibit drastic changes of transmittances between $25^{\circ} \mathrm{C}$ and $80^{\circ} \mathrm{C}$ (Figure $1 \mathrm{~b}$ and $1 \mathrm{c}$ ). Because the motion of the polymer chains in $\mathbf{2 b}, \mathbf{c}$ was restricted due to high contents of cross-linking points, they could not freely change between aggregation and dispersion states in hydrogels depending on temperatures as those were observed in 2a. These results indicate that

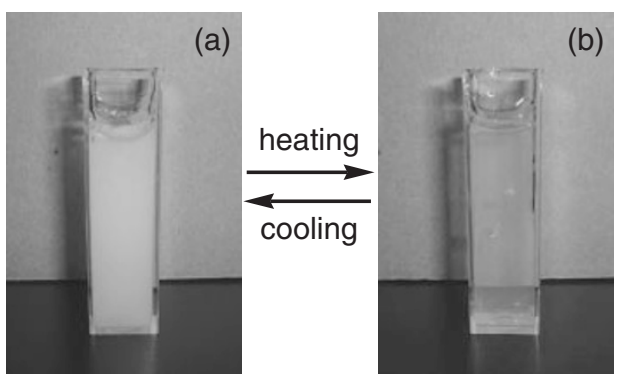

Figure 2. Photographs of $\mathbf{2 a}$ at $25^{\circ} \mathrm{C}$ (a) and $80^{\circ} \mathrm{C}$ (b).

the chemical cross-linking densities in the hybrid hydrogels are important factor for their temperatureresponse. Furthermore, the hydrogels 3a-c, which were synthesized using BisAAm as a conventional organic cross-linking agent and MAAm with different feed molar ratios ([BisAAm]/[MAAm $]=0.5(3 \mathbf{a})$, $1.0(\mathbf{3 b})$, and $3.0 \%(3 \mathbf{c}))$, did not exhibit drastic tem- 


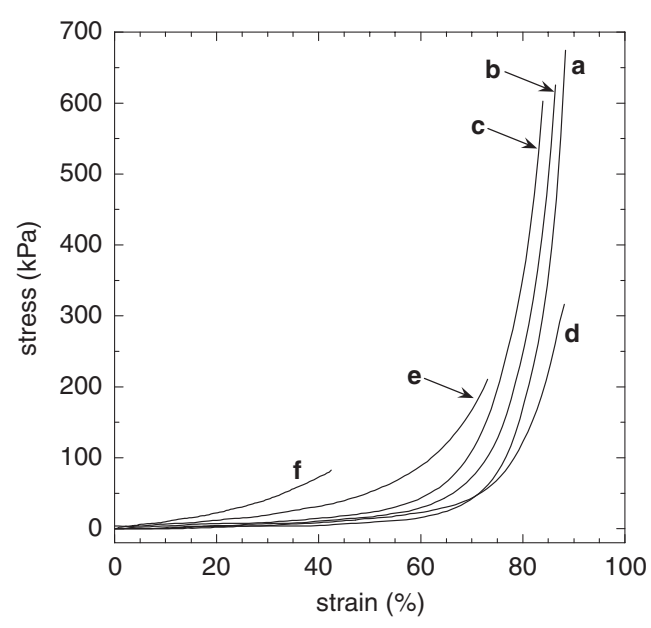

Figure 3. Stress-strain curves under compression for hybrid hydrogels $\mathbf{2 a}$ (a), $\mathbf{2 b}$ (b), 2c (c) and hydrogels $\mathbf{3 a}$ (d), $\mathbf{3 b}$ (e), $\mathbf{3 c}$ (f).

perature-response (Figure 1d-1f). These results probably indicate that the polysiloxane-based cross-linking agent 1 specifically affected the temperature-responsive behavior of PMAAm hydrogel.

Figure 3 shows stress-strain curves under compression of the hybrid hydrogels 2 and PMAAm hydrogels 3. The hydrogels 3 broke at stresses in the range of 83-317 kPa (Figure 3d-3f), while the hybrid hydrogels 2 sustained the stresses in the range of 603$675 \mathrm{kPa}$ and fracture strains of 2 were $84-88 \%$ (Figure 3a-3c). These results indicate that the compressive properties of the hybrid hydrogels 2 are more superior than those of the hydrogels $\mathbf{3}$ obtained using conventional organic cross-linking agent, probably because that the cross-linking points were dispersed well in the hydrogel 2 . Such a tendency was also observed with the hydrogels obtained using AAm monomer in our previous study. ${ }^{11}$

\section{CONCLUSION}

We have prepared temperature-responsive hybrid hydrogels by free-radical polymerization of MAAm using water-soluble rigid polysiloxane having acrylamido side-chains as a cross-linking agent. The hybrid hydrogel obtained using the polysiloxane-based crosslinking agent with relative low functionality of acrylamido group showed obvious temperature-response, i.e., it was turbid at $25^{\circ} \mathrm{C}$ and almost transparent at $80^{\circ} \mathrm{C}$. The compressive properties of the hybrid hydrogels were more superior than those of PMAAm hydrogels obtained using conventional organic crosslinking agent.

Acknowledgment. Y.K. acknowledges financial support from The Asahi Glass Foundation.

\section{REFERENCES}

1. "Gels Handbook," Y. Osada and K. Kajiwara, Ed., Academic Press, 2001.

2. L. E. Bromberg and E. S. Ron, Adv. Drug Delivery Rev., 31, 197 (1998).

3. Y. Qiu and K. Park, Adv. Drug Delivery Rev., 53, 321 (2001).

4. Y. Kaneko, S. Nakamura, K. Sakai, T. Aoyagi, A. Kikuchi, Y. Sakurai, and T. Okano, Macromolecules, 31, 6099 (1998).

5. J. Zhang and N. A. Peppas, Macromolecules, 33, 102 (2000).

6. H. K. Ju, S. Y. Kim, and Y. M. Lee, Polymer, 42, 6851 (2001).

7. K. Haraguchi and T. Takehisa, Adv. Mater., 14, 1120 (2002).

8. K. Haraguchi, H.-J. Li, K. Matsuda, T. Takehisa, and E. Elliott, Macromolecules, 38, 3482 (2005).

9. Y. Kaneko, N. Iyi, K. Kurashima, T. Matsumoto, T. Fujita, and K. Kitamura, Chem. Mater., 16, 3417 (2004).

10. Y. Kaneko, N. Iyi, T. Matsumoto, and K. Kitamura, Polymer, 46, 1828 (2005).

11. Y. Kaneko, S. Sato, J. Kadokawa, and N. Iyi, J. Mater. Chem., 16, 1746 (2006).

12. Y. Kaneko, J. Kadokawa, M. Setoguchi, and N. Iyi, Polymer, 46, 8905 (2005). 